Esta revista forma parte del acervo de la Biblioteca Jurídica Virtual del Instituto de Investigaciones Jurídicas de la UNAM

\title{
El modelo de comunicación política: problemas de aplicabilidad, coordinación e interpretación en el ámbito federal y estatal
}

\section{Citlali Villafranco Robles* Luis Eduardo Medina Torres**}

\section{Sumario:}

I. Introducción

II. Consideraciones metodológicas

III. Hallazgos centrales

IV. El modelo de comunicación política: aplicación de la justicia electoral

V. Propuestas para posibles reformas al régimen electoral VI. Fuentes consultadas

* Profesora investigadora en la Universidad Autónoma de la Ciudad de México; miembro del Sistema Nacional de Investigadores; posdoctora en el Posgrado de Estudios Sociales, en la línea de Estudios Políticos de la Universidad Autónoma Metropolitana Iztapalapa.

** Profesor-investigador en la Universidad Autónoma de la Ciudad de México, con licencia al cargo; miembro del Sistema Nacional de Investigadores nivel I.

D. R. () 2013. PNUD-UNAM, Instituto de Investigaciones Jurídicas. Revista Mexicana de Derecho Electoral, Especial sobre Observación Electoral 2012, 
Esta revista forma parte del acervo de la Biblioteca Jurídica Virtual del Instituto de Investigaciones Jurídicas de la UNAM www.juridicas.unam.mx

\section{Resumen:}

El presente trabajo aborda aspectos de la reforma electoral de 1997, así como sobre su aplicación y las dificultades presentadas en las elecciones de 2006, para de esta manera llegar a la reforma de 2007, con la cual se hizo frente a la elección presidencial de 2012.

El punto medular de esta investigación versa en el modelo de comunicación política y los derechos en disputa, como lo son: libertad de expresión y de información, de tal suerte que se analizan el desenvolvimiento de las autoridades al ponderar dichos derechos y el sentido de sus resoluciones.

Se encuentra dividida en tres partes, aborda aspectos metodológicos, los resultados en opinión de los autores sobre la aplicación del modelo de comunicación política, para finalizar con un apartado de propuestas.

Palabras clave: autoridades electorales, modelo de comunicación política, derecho a la información, libertad de expresión, justicia electoral, propaganda. 


\section{Introducción}

Con la reforma electoral de 1997 el país dio paso a la alternancia política. Un avance extraordinario consistió en la construcción de una institucionalidad democrática que permitiera la sustitución del partido de Estado y reconociera plenamente la existencia de un sistema de partidos. Sin embargo, en 2006 esta reforma mostró límites importantes que provocaron que el resultado de la elección presidencial fuera cuestionado por una parte significativa de la ciudadanía.

En el centro de este cuestionamiento estaba el peso indebido ganado por las televisoras privadas, como resultado de un modelo de comunicación político-electoral en el que los partidos libremente contrataban tiempo en radio y televisión, con la restricción relativa de los recursos presupuestales y los topes de gasto en las campañas. Las televisoras, por su parte, de común acuerdo con la autoridad electoral establecían los precios máximos por los tiempos de transmisión, pero se reservaban los descuentos aplicables. Consecuentemente, sus preferencias políticas se traducían en precios diferenciales para los distintos partidos y candidatos.

La crisis de 2006 dio lugar a una nueva reforma constitucional, publicada en 2007, en la que se modificó sustancialmente el modelo de comunicación política en el proceso electoral. Al prohibirse la contratación directa de tiempo en radio y televisión y resolver que los únicos espacios disponibles serían los administrados por el Instituto Federal Electoral (IFE), el Legislativo decidió privilegiar el derecho a la información sobre la libertad de expresión que tutelaba prioritariamente la legislación de 1997.

La aplicación de la nueva normatividad electoral, desde su inicio, estuvo marcada por cuestionamientos centrados precisamente en este dilema entre derechos. Su primera prueba, las elecciones intermedias de 2009, mostraron dificultades de aplicación y diferencias de interpretación del modelo de comunicación diseñado por el legislador. La prueba mayor era, por supuesto, la elección presidencial de 2012.

Investigar estas dificultades en todo el proceso electoral de 20112012 resultaba de enorme importancia. A continuación se presentan los resultados de la investigación sobre la aplicación del nuevo modelo de comunicación política y de las distintas interpretaciones vertidas 
Esta revista forma parte del acervo de la Biblioteca Jurídica Virtual del Instituto de Investigaciones Jurídicas de la UNAM

por las autoridades electorales, el IFE y el Tribunal Electoral del Poder Judicial de la Federación (TEPJF). El orden de presentación de estos resultados es el siguiente: en la primera parte se ofrecen las consideraciones metodológicas, las preguntas y las hipótesis fundamentales con las que se trabajó; la segunda parte presenta los hallazgos centrales del trabajo; en la tercera parte se ofrece una apreciación global de la aplicación de la justicia electoral en relación con el modelo de comunicación; finalmente, se plantean algunas propuestas para posibles reformas del régimen electoral en las áreas revisadas.

\section{Consideraciones metodológicas}

Para desarrollar esta investigación, decidimos centrarnos en los alcances y límites de la aplicación de la reforma electoral en lo correspondiente a los medios de comunicación, consecuentemente, definimos revisar los acuerdos y resoluciones del IFE en el periodo que comprende de octubre de 2011 a septiembre de 2012, en lo que correspondiera a procedimientos administrativos y sanciones relativas a la difusión de propaganda en medios de comunicación.

Como estas determinaciones de la autoridad administrativa pueden ser impugnadas ante las salas del TEPJF, incorporamos al análisis las sentencias de ese órgano jurisdiccional relacionadas con los medios de comunicación, ya fueran de ámbito nacional o local.

En un primer análisis, se revisaron todos los acuerdos y resoluciones emitidas por el IFE en el periodo de estudio, así como todas las sentencias del TEPJF. Por razones metodológicas, fueron seleccionados solamente los documentos correspondientes al modelo de comunicación política, de modo tal que temas como el rebase de topes de gastos de campaña, el uso publicitario de las encuestas o los delitos electorales, se abordan indirectamente en la investigación por no ser su objeto de estudio.

Los cortes metodológicos señalados, explican que en este trabajo no se revisen los resultados electorales, salvo cuando es necesario hacer alguna referencia o remisión puntual a los votos, como sucedió con la sentencia que resolvió la impugnación general de la elección presidencial (SUP-JIN-359/2012). Por las mismas razones, tampoco se analizan los casos de investigaciones por delitos electorales o por 
Esta revista forma parte del acervo de la Biblioteca Jurídica Virtual del Instituto de Investigaciones Jurídicas de la UNAM

faltas administrativas, salvo cuando hayan estado relacionadas directamente con los medios de comunicación.

Para contar con un análisis completo de estas interpretaciones de la legislación emitida por el Poder Legislativo, incluimos todas las fases del proceso electoral. Por esto, el periodo de investigación que inicialmente comprendería doce meses, fue reducido a once ya que el proceso federal concluyó el 31 de agosto de 2012 con la calificación de la elección presidencial. La cobertura geográfica observada fue todo el país, ya que las impugnaciones se presentan en los 300 consejos distritales del IFE, en sus 32 consejos locales, en el Consejo General, así como en las cinco salas regionales o en la Sala Superior del TEPJF.

Para delimitar el universo observable, estipulamos una serie de variables que permitieron discriminar los documentos (acuerdos, resoluciones o sentencias) que correspondieran al objeto de estudio, es decir, al modelo de comunicación política y las interpretaciones que realizaron las autoridades electorales. Además, fijamos nuestra atención en los casos en que hubo discrepancia entre el IFE y el TEPJF, ya fuera por el criterio de aplicación o por el derecho que debería ser tutelado en el caso impugnado.

La sistematización está contenida en una base de registros que contemplan las variables indicadas en el proyecto; de las que destacan:

- Clave, que referencia acuerdos y resoluciones con sentencias.

- Resumen de los votos, que informa las consideraciones y el resultado de acuerdos, resoluciones y sentencias.

- Votos particulares, que revela algún disenso en las votaciones de consejeros y magistrados.

- Derecho garantizado, que ubica acuerdos, resoluciones y sentencias que tutelan la libertad de expresión o el derecho a la información.

- Contradicción de criterios, que se refiere a las diferencias entre las dos autoridades electorales acerca de algún litigio en concreto, lo que permitió construir el evaluador del modelo de comunicación política.

Con la investigación pretendimos dar respuesta a dos preguntas centrales: ¿cómo se aplicó el modelo de comunicación política por las autoridades electorales nacionales?, y ¿cómo interpretaron esas autoridades las regulaciones contenidas en la legislación electoral a la luz 
Esta revista forma parte del acervo de la Biblioteca Jurídica Virtual del Instituto de Investigaciones Jurídicas de la UNAM

de sus resoluciones, ya fueran de carácter administrativo o de orden jurisdiccional.

La hipótesis central de la investigación fue que las autoridades electorales, en un ejercicio de interpretación y de adecuación, han modificado al modelo de comunicación política.

\section{Hallazgos centrales}

La respuesta a las preguntas “¡cómo interpretan y aplican el modelo de comunicación política los órganos electorales federales?” y “ ¿xisten contradicciones entre los criterios de interpretación del IFE y del TEPJF?" influyeron para que el modelo de comunicación política decidido por los legisladores en 2008 fuera modificado por las autoridades electorales, quienes interpretaron y armonizaron las disposiciones legales.

En ese ejercicio interpretativo, las autoridades tuvieron que tomar una decisión acerca de la salvaguarda de derechos fundamentales, y en este sentido hubo una preocupación mayor por la libertad de expresión. Un segundo hallazgo consiste en el acuerdo que existió entre ambas autoridades en la mayoría de los casos.

Es de resaltar que, en un ejercicio dialógico, pareciera que los órganos electorales nacionales tomaron una decisión acerca del bien jurídico por tutelar, esto es, la libertad de expresión; empero, la legislación electoral estipuló en la reforma de 2007-2008 que la equidad en la contienda y el derecho a la información eran los propósitos a conseguir, debido a las intervenciones de actores no electorales en los comicios presidenciales de 2006.

Tutelar el principio y derecho que comentamos fue el propósito de la reforma electoral más reciente, el problema es que con un ejercicio interpretativo como el realizado en los comicios federales de 2012, las autoridades electorales transformaron de manera notable el modelo de comunicación política, estableciendo uno menos restrictivo que el planteado en la legislación electoral.

En el periodo de análisis ambas instancias federales emitieron 456 acuerdos, resoluciones y sentencias relacionadas con los medios de comunicación. El IFE concentró el 70\% de ellos, con 318 acuerdos y 
Esta revista forma parte del acervo de la Biblioteca Jurídica Virtual del Instituto de Investigaciones Jurídicas de la UNAM

resoluciones, mientras que el TEPJF emitió el 30\% del total con 138 sentencias.

Un aspecto relevante de estas determinaciones es que el $49 \%$ fueron favorables a la libertad de expresión, en tanto que el $43 \%$ lo fueron al derecho a la información.

Tabla 1. Derecho garantizado

\begin{tabular}{|c|c|c|}
\hline Derecho garantizado & Número & Porcentaje \\
\hline Derecho a la información & 198 & 43.42 \\
\hline Libertad de expresión & 224 & 49.12 \\
\hline No aplica & 34 & 7.46 \\
\hline Total & 456 & 100.00 \\
\hline
\end{tabular}

Fuente: elaboración propia a partir de la base de datos electoral PNUDUACM.

La preocupación de las autoridades electorales por tutelar la libertad de expresión resulta más relevante cuando en las quejas se involucra a los medios de comunicación, por ejemplo, la sentencia del TEPJF del 1 de marzo de 2012 con la que confirmó el acuerdo del IFE que tuvo por infundado el procedimiento especial sancionador contra Enrique Peña Nieto, quien en su carácter de aspirante y precandidato del Partido Revolucionario Institucional a la Presidencia de la República, participó en diversos actos públicos que fueron reseñados en notas informativas, periodísticas o noticiosas emitidas a través de prensa escrita, Internet y televisión. La Sala argumentó que los medios de comunicación que dieron a conocer dichos hechos únicamente estaban cumpliendo su deber de informar con la debida capacidad de presentar cualquier suceso (SUP-RAP-041/2012).

Lo anterior es uno de los hallazgos importantes de nuestra investigación. El modelo definido por el legislador contemplaba una mayor protección del derecho a la información, mientras que la interpretación y aplicación del órgano administrativo y del órgano jurisdiccional protegía la libertad de expresión. Esta conclusión sobre la redefinición del modelo de comunicación adquiere mayor solidez cuando se toma en cuenta que el $88 \%$ de las resoluciones fueron votadas por unanimidad. 
Esta revista forma parte del acervo de la Biblioteca Jurídica Virtual del Instituto de Investigaciones Jurídicas de la UNAM

La elección sobre la que se realizan el mayor número de demandas es, por supuesto, la elección presidencial con el 59\% de las quejas, en tanto que las elecciones para gobernadores y Congresos estatales ocupan el segundo lugar con el 16\% de los casos.

Tabla 2. Tipo de elección

\begin{tabular}{|c|c|c|}
\hline Tipo de elección & Número & Porcentaje \\
\hline Presidencial & 267 & 58.55 \\
\hline Senadores & 23 & 5.04 \\
\hline Diputados & 58 & 12.72 \\
\hline Estatal & 72 & 15.79 \\
\hline Municipal & 3 & 0.66 \\
\hline No aplica & 33 & 7.24 \\
\hline Total & 456 & 100.00 \\
\hline
\end{tabular}

Fuente: elaboración propia a partir de la base de datos electoral PNUDUACM.

Los medios de comunicación fueron los grandes protagonistas de las demandas por la propaganda que se difundió en radio y televisión con el 59\%.

Tabla 3. Tipo de medio de comunicación

\begin{tabular}{|c|c|c|}
\hline Tipo de medio de comunicación & Número & Porcentaje \\
\hline Alternativo & 45 & 9.87 \\
\hline Impreso & 64 & 14.04 \\
\hline Radio y TV & 164 & 35.96 \\
\hline Radio & 44 & 9.65 \\
\hline Televisión & 61 & 13.38 \\
\hline Varios & 59 & 12.94 \\
\hline No aplica & 19 & 4.17 \\
\hline Total & 456 & 100.00 \\
\hline
\end{tabular}

Fuente: elaboración propia a partir de la base de datos electoral PNUDUACM. 
Esta revista forma parte del acervo de la Biblioteca Jurídica Virtual del Instituto de Investigaciones Jurídicas de la UNAM

En cuanto al tipo de acto demandado, los reclamos se concentran en la propaganda electoral con el 59\% de los casos, es decir, en los mensajes o información en que se presenta un partido o candidato y se pide el voto. Un dato relevante es que el $22 \%$ de los reclamos son por propaganda gubernamental, lo que habla de la intervención de los gobiernos de los diferentes niveles alterando las condiciones de competencia. La propaganda política apenas alcanza un $11 \%$, lo que muestra que en este ámbito las restricciones han sido respetadas, aunque ha habido casos polémicos de deportistas, artistas o comunicadores.

Tabla 4. Tipo de propaganda

\begin{tabular}{|c|c|c|}
\hline Tipo de propaganda & Número & Porcentaje \\
\hline Electoral & 268 & 58.77 \\
\hline Gubernamental & 100 & 21.93 \\
\hline Politico & 61 & 13.38 \\
\hline No aplica & 27 & 5.92 \\
\hline Total & 456 & 100.00 \\
\hline
\end{tabular}

Fuente: elaboración propia a partir de la base de datos electoral PNUDUACM.

Los mayores demandantes son los partidos políticos, quienes interpusieron el 66\% de las impugnaciones y el mayor quejoso fue el PRI, quien en el pasado proceso electoral interpuso el $24 \%$ de las quejas.

La característica de nuestro sistema electoral, que tiene como protagonistas a los partidos políticos, explica que sean éstos los que más demandan y también los más demandados, y en este tenor, durante el proceso electoral federal de 2012 el partido político más demandado fue Acción Nacional, quien concentró el 10\% de las demandas.

Encontramos que de las 138 sentencias emitidas por el TEPJF, en el $62 \%$ de los casos confirmaron las resoluciones del IFE y en 7\% las modificaron. De este modo, en casi el 70\% de las sentencias el Tribunal Electoral y el IFE aplicaron los mismos criterios. En el 30\% restante el TEPJF revocó las resoluciones del IFE. 
Esta revista forma parte del acervo de la Biblioteca Jurídica Virtual del Instituto de Investigaciones Jurídicas de la UNAM

Tabla 5. Sentido de las sentencias respecto a los acuerdos y las resoluciones del IFE

\begin{tabular}{|c|c|c|}
\hline Sentido de las sentencias & Número & Porcentaje \\
\hline Confirma & 86 & 62.32 \\
\hline Modifica & 9 & 6.52 \\
\hline Multa & 0 & 0.00 \\
\hline Revoca & 41 & 29.71 \\
\hline No aplica & 2 & 1.45 \\
\hline Total & 138 & 100.00 \\
\hline
\end{tabular}

Fuente: elaboración propia a partir de la base de datos electoral PNUDUACM.

Uno de los obstáculos del actual modelo de comunicación política es su aplicación, pues es un modelo que limita el acceso de los partidos políticos a los medios de comunicación, en tanto que estos actores de interés público, están altamente interesados en ocupar espacios en los medios. El modelo también restringe la participación de actores económicos y particulares, quienes de igual forma tienen interés en intervenir en los procesos electorales, básicamente durante los periodos de campañas

Para incentivar el cumplimiento de la norma se requieren sanciones muy altas; sin embargo, es justamente en la aplicación de los castigos donde se encuentra una de las principales debilidades de las autoridades electorales federales pues solamente en el 11\% de los casos impusieron una multa, mientras que en el $5 \%$ impusieron una amonestación.

Tabla 6. Tipo de sanción aplicada

\begin{tabular}{|c|c|c|}
\hline Tipo de sanción & Número & Porcentaje \\
\hline Amonestación & 23 & 5.04 \\
\hline Confirma & 86 & 18.86 \\
\hline Modifica & 9 & 1.97 \\
\hline Multa & 49 & 10.75 \\
\hline Revoca & 41 & 8.99 \\
\hline No aplica & 248 & 54.39 \\
\hline Total & 456 & 100.00 \\
\hline
\end{tabular}

Fuente: elaboración propia a partir de la base de datos electoral PNUDUACM. 
La interpretación que realizaron las autoridades electorales en materia de medios de comunicación fue el resultado de un esfuerzo de ponderación y adecuación. Sin embargo, las divergencias principales se advierten en el alcance y sentido de las restricciones, ya que al interpretar el régimen jurídico se privilegió el derecho a la información o la libertad de expresión.

Tales diferendos significan, además, el establecimiento de precedentes que serán aplicados en ocasiones posteriores y revelan el comportamiento de los partidos que cuando impugnaban exigían una aplicación estricta, mientras que si eran demandados pedían una aplicación laxa de la ley.

\section{El modelo de comunicación política: aplicación de la justicia electoral}

Previo al proceso electoral de 2011-2012 hubo modificaciones constitucionales y legales que dieron nuevas atribuciones a las autoridades electorales. En el caso del IFE se incorporó un procedimiento especial sancionador (PES), para el dictado de medidas cautelares, y en el ámbito procesal se ampliaron los sujetos obligados, además, se incorporó a los ciudadanos como posibles demandantes de los recursos de apelación, particularmente por el tema de las posibles violaciones al régimen electoral.

Con estas nuevas disposiciones, las dos autoridades federales en la arena electoral tuvieron que hacer frente a procedimientos administrativos y a procesos jurisdiccionales por lo que fueron adecuando las normas electorales contenidas en el Código Federal de Instituciones y Procedimientos Electorales y en la Ley General del Sistema de Medios de Impugnación.

En términos de justicia electoral, las sentencias derivadas de los recursos de apelación en la órbita federal, así como los juicios de revisión constitucional terminaron en la esfera de las salas del TEPJF durante el proceso electoral federal reciente (octubre de 2011 a agosto de 2012), fueron 138 relativas al tema de medios de comunicación, mensajes de propaganda electoral, de propaganda gubernamental y de comunicación política. 
Esta revista forma parte del acervo de la Biblioteca Jurídica Virtual del Instituto de Investigaciones Jurídicas de la UNAM

Los datos de la tabla 4 arrojan que, además de los conflictos entre los partidos por propaganda en las elecciones, también existen intentos de intervención de los diversos órdenes de gobierno y se suman a ello, los casos de ciudadanos por propaganda política en elecciones.

De las 138 sentencias, 86 resoluciones confirmaron los acuerdos y las determinaciones del IFE; 41 sentencias revocaron sus resoluciones y 9 las modificaron. Buena parte de las revocaciones estuvieron relacionadas con la individualización de la sanción.

Si bien la mayoría de acuerdos y resoluciones del IFE fueron confirmadas, también es cierto que, en lo relativo a las sanciones y a la interpretación de derechos fundamentales, existe un conflicto de perspectivas entre las dos autoridades electorales como se muestra a continuación.

Tabla 7. Criterios sobre libertad de expresión y derecho a la información del TEPJF

\begin{tabular}{|c|c|c|}
\hline Derecho garantizado & Número & Porcentaje \\
\hline Derecho a la Información & 73 & $52.90 \%$ \\
\hline Libertad de Expresión & 65 & $47.10 \%$ \\
\hline Total & 138 & $100.00 \%$ \\
\hline
\end{tabular}

Fuente: elaboración propia a partir de la base de datos electoral PNUDUACM.

De las 138 sentencias del TEPJF, en 65\% de los casos avaló criterios relativos a la libertad de expresión, mientras que en $73 \%$ sostuvo el criterio de derecho a la información. El punto es que el modelo de comunicación política incorpora restricciones a favor del derecho a la información, por lo que su protección debió ser más amplia.

Respecto a las votaciones de los magistrados electorales, cabe decir que 122 casos fueron resueltos por unanimidad, mientras que en 16 sentencias hubo diferendo entre los magistrados, y se resolvieron por mitad, es decir, hacia la protección del derecho a la información o bien, de la libertad de expresión, lo que es consistente con los datos agregados del total de sentencias en el periodo estudiado (52.90\% frente a $47.10 \%$, véase tabla 7 ). 
Los datos iniciales de la investigación muestran que hay una discusión institucional en la orientación y la interpretación del modelo de comunicación, ya que han existido disensiones entre los aplicadores de las normas.

Estos mismos datos respaldan de manera inicial la hipótesis central de la investigación en el sentido de que las autoridades electorales han modificado con sus interpretaciones el modelo de comunicación política, lo que ha generado más dificultades en relación con los problemas que han intentado resolver.

Nuestro propósito fue revisar hasta qué punto las interpretaciones modificaron el modelo de comunicación. La principal conclusión de nuestro trabajo es que, del universo de registros estudiados podemos desprender que ha existido una tendencia favorable a la aplicación de reglas comunes entre el IFE y el TEPJF, la que se aleja del modelo diseñado por el legislador - que privilegiaba el derecho a la información-.

Una segunda conclusión deriva del periodo de análisis, en el cual se presentaron casos que sometieron a prueba el modelo de comunicación y evidenciaron la baja cooperación y cumplimiento de las normas por parte de los protagonistas del proceso electoral, así como de los funcionarios públicos que realizaron propaganda gubernamental.

Los partidos políticos mostraron poco interés en cumplir las normas en materia de medios de comunicación, y por el contrario buscaron oportunidades para esquivarlas. Este desapego hacia la norma no se resuelve con nuevos reglamentos, porque en materia de medios de comunicación estamos sobrerreglamentados, se trata de un problema que deriva del comportamiento de los actores y sólo se resolverá modificando su conducta.

\section{Propuestas para posibles reformas al régimen electoral}

El modelo de comunicación política establecido en la reforma electoral de 2007-2008, presenta algunos problemas de aplicación que fueron detectados con la investigación realizada. A continuación se enuncian algunos caminos para discutir su posible solución:

Es propicio alentar el régimen sancionador hacia una posible legislación de partidos políticos diversa a la ley electoral, puesto que 
Esta revista forma parte del acervo de la Biblioteca Jurídica Virtual del Instituto de Investigaciones Jurídicas de la UNAM

esa norma comprendería un catálogo completo de sanciones que hoy en día se localizan en los reglamentos del IFE y otros en el código electoral. Es decir, habría una condensación de faltas administrativas, sanciones y aplicabilidad con un solo criterio.

De lo anterior se desprende la incorporación de elementos regulatorios en ámbitos diversos a la materia electoral, es decir, propiciar que algunos preceptos se instituyan en otros marcos regulatorios para incentivar que los partidos políticos u otros actores asuman con seriedad y responsabilidad el régimen legal.

En este sentido, también es imperante homologar el marco regulatorio tanto de la Ley Federal de Radio y Televisión y del Código Federal de Instituciones y Procedimientos Electorales, con el objetivo de que los concesionarios y permisionarios de los medios de comunicación atiendan con seriedad y responsabilidad las sanciones que en materia electoral se les imputen. En este mismo orden, hacer competente a la Secretaría de Comunicaciones y Transportes, para la aplicación de sanciones relativas a la materia electoral.

Es indispensable definir con precisión en la legislación electoral los alcances de dos derechos fundamentales: libertad de expresión y acceso a la información pública, a efecto de que los actores involucrados no aprovechen los espacios de indefinición.

Es necesario discutir la reglamentación, en las leyes de responsabilidades de los servidores públicos, tanto en el ámbito federal como a nivel de las entidades federativas, y de sanciones y prohibiciones relativas al artículo 134 de la Constitución Política de los Estados Unidos Mexicanos concernientes a la prohibición de transmitir publicidad gubernamental en procesos electorales, así como sobre la propaganda personalizada.

Es conveniente perfeccionar la instrumentación de mecanismos de control por parte de la autoridad electoral, que permitan verificar que no existe compra de publicidad disfrazada por parte de sujetos que prohíbe la ley.

\section{Fuentes consultadas}

Base de datos del proyecto PNUD-UACM.

Sentencia SUP-RAP-041/2012. Actor: Partido Acción Nacional. Autoridad Responsable: Consejo General del Instituto Federal Elec- 
Esta revista forma parte del acervo de la Biblioteca Jurídica Virtual del Instituto de Investigaciones Jurídicas de la UNAM

toral. Disponible en $w w w . t e . g o b . m x$, consultada el 10 de diciembre del 2012.

Sentencia SUP-JIN-359/2012. Actor: Coalición Movimiento Progresista. Autoridad Responsable: Consejo General del Instituto Federal Electoral. Disponible en www.te.gob.mx, consultada el 5 de diciembre del 2012. 